\section{Influência da dor no controle postural de mulheres com dor cervical}

\author{
Influence of pain on postural control in women with \\ neck pain
}

\author{
Juliana Corrêa Soares ${ }^{1}$ \\ Priscila Weber ${ }^{1}$ \\ Maria Elaine Trevisan ${ }^{2}$ \\ Claudia Morais Trevisan ${ }^{3}$ \\ Carlos Bolli Mota ${ }^{4}$ \\ Angela Garcia Rossi ${ }^{5}$
}

Resumo - O objetivo foi verificar a influência da dor no controle postural de mulheres com dor cervical e a relação com as possíveis alterações nos sistemas sensoriais e postura corporal. O grupo dor cervical foi composto por mulheres, entre 20 e 50 anos, com dor cervical por mais de três meses e o grupo controle por 20 mulheres sem dor cervical. Para caracterização, utilizaram-se anamnese, índice de incapacidade cervical e Escala Visual Analógica. O equilíbrio postural foi avaliado por uma plataforma de força. O equilíbrio com manipulação dos sistemas sensoriais foi avaliado pela posturografia dinâmica Foam-laser, expondo o indivíduo a seis testes de organização sensorial. A postura foi avaliada pelo Software de Avaliação Postural. Normalidade das variáveis verificada pelo teste de Shapiro Wilk e testes $t$ de student e Mann Whitney para comparação entre grupos, nível de significância de $5 \%$. Os grupos apresentaram homogeneidade nas variáveis demográficas. No equilíbrio postural, observou-se maior amplitude e velocidade de deslocamento do centro de pressão no grupo dor cervical, demonstrando maior oscilação postural. Houve diferença significativa no ângulo crâniovertebral, mostrando anteriorização da cabeça nas mulheres sintomáticas. Na posturografia dinâmica, observou-se diferença entre os grupos sendo que o escore obtido nas seis condições sensoriais demonstrou que o grupo dor cervical apresentou maior comprometimento do equilíbrio. Dor cervical e postura anteriorizada da cabeça têm efeito deletério no controle postural de mulheres sintomáticas, tanto na postura estática quanto na postura dinâmica.

Palavras-chave: Cervicalgia; Equilíbrio postural; Postura; Propriocepção; Sistema vestibular.

Abstract - The objective of this study was to investigate the influence of pain on postural control in women with neck pain and the relationship with possible changes in sensory systems and posture. The neck pain group was composed of women, aged between 20 and 50 years, complaining of neck pain for more than three months; the control group was composed of women without complaints of neck pain. For the characterization of the groups, we used anamnesis, neck disability index and Visual Analogue Scale. Postural balance was assessed on force platform. Postural balance with manipulation of the sensory systems was measured by Foam Laser Dynamic Posturography, exposing the individual to six sensory organization tests. Posture was assessed by the Postural Assessment Software. The normality of the variables were verified using Shapiro-Wilk test, Student's t-test and Mann-Whitney test for comparison between groups, with a significance level of $5 \%$. Groups were homogeneous in demographic variables. We observed higher amplitude and displacement velocity of the center of pressure in the neck pain group, showing greater postural balance. There were significant differences in craniovertebral angle, showing forward head posture in symptomatic women. In dynamic posturography, we observed a difference between the groups: the score obtained in the six sensory conditions showed that neck pain group presented greater balance impairment. Neck pain and forward head posture have a deleterious effect on postural control in symptomatic women, both in the static posture and dynamic posture.

Key words: Labyrinth; Neck pain; Postural balance; Posture; Proprioception; Vestibule.
1 Universidade Federal de Santa Maria. Programa de Pós-graduação (Mestrado) em Distúrbios da Comunicação Humana. Santa Maria, RS. Brasil.

2 Universidade Federal de Santa Maria. Departamento de Fisioterapia e Reabilitação. Programa de Pós-graduação (Doutorado) em Distúrbios da Comunicação Humana. Santa Maria, RS. Brasil.

3 Universidade Federal de Santa Maria. Departamento de Fisioterapia e Reabilitação. Santa Maria, RS. Brasil.

4. Universidade Federal de Santa Maria. Departamento de Métodos e Técnicas Desportivas. Programa de Pós-graduação em Educação Física. Santa Maria, RS. Brasil.

5. Universidade Federal de Santa Maria. Departamento de Fonoaudiologia. Programa de Pós-graduação em Distúrbios da Comunicação Humana. Santa Maria, RS. Brasil.

Recebido em 06/08/12 Revisado em 19/10/12 Aprovado em 05/12/12

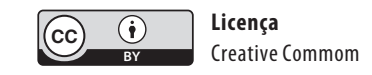




\section{INTRODUÇÃO}

A dor na região cervical vem sendo considerada um dos mais onerosos problemas osteomusculares, com enorme impacto sobre a saúde e qualidade de vida dos indivíduos e na sociedade como um todo ${ }^{1}$. Esta disfunção vem aumentando consideravelmente nos últimos anos. Estima-se que, aproximadamente, $50 \%$ dos indivíduos adultos experimentarão dor cervical em algum momento da vida ${ }^{2}$, sendo que $75 \%$ destes terão recorrência da dor nos próximos cinco anos ${ }^{3}$.

Recentemente, a dor na região cervical tem sido amplamente classificada como de origem idiopática ou induzida por um trauma na região cervical como, por exemplo, lesão de chicote, não sendo possível a realização de um diagnóstico anatomopatológico definitivo na maioria dos casos. Essa classificação reconhece a diferença no mecanismo insidioso da dor, podendo haver diferença na natureza e nas caracteristicas fisiopatológicas entre os dois tipos de dor na região cervical ${ }^{4}$.

Nas disfunções cervicais, a dor é geralmente o sintoma mais frequente, estando relacionada aos esforços repetitivos e a manutenção de posturas inadequadas durante a atividade ocupacional ${ }^{5}$, que acarretam microtraumatismos às vértebras cervicais e aos tecidos moles periarticulares ${ }^{6,7}$. A literatura sugere a influência da dor cervical sobre o controle postural em decorrência de alterações na entrada somatossensorial cervical e integração do sistema de controle postural ${ }^{4}$. O efeito da dor sobre os nociceptores e mecanorreceptores, influencia a modulação central da aferência e, consequentemente, afeta o controle neuromuscular e postural, resultando em aumento da oscilação postural, sensação de instabilidade, tonturas, náuseas ou alterações visuais ${ }^{8,9}$.

A manutenção do equilíbrio na postura ereta ocorre por meio da atuação do controle postural, que obtém informações sensoriais do sistema visual, vestibular e somatossensorial ${ }^{10,11}$. Uma das tarefas mais importantes do sistema de controle postural é a manutenção do equilíbrio sob a base de apoio fornecida pelos pés. Essa manutenção inclui a detecção sensorial dos movimentos corporais, a integração das informações sensório-motoras no Sistema Nervoso Central (SNC) e a execução das respostas musculoesqueléticas apropriadas. Qualquer comprometimento desse complexo sistema de integração pode dar origem ao desequilíbrio corporal ${ }^{10,12}$.

A maneira mais utilizada para estudar o controle postural é a avaliação da oscilação do centro de pressão (COP) durante a manutenção da postura ereta quieta ${ }^{11}$. A técnica utilizada para medir a oscilação do corpo ou de uma variável associada é a posturografia, comumente dividida em posturografia estática, quando a postura ereta quieta do sujeito é estudada e posturografia dinâmica, quando a resposta a uma perturbação aplicada sobre o sujeito é objeto de estudo ${ }^{11}$.

Dada a complexidade biomecânica envolvida na manutenção do equilíbrio corporal e alinhamento postural, nota-se que, diversos fatores podem intervir de forma negativa, provocando ajustes corporais e motores 
compensatórios. Alterações na condução, integração ou processamento das informações sensoriais causadas pelas alterações posturais podem convergir para um equilíbrio corporal menos eficiente. Acredita-se que a dor cervical pode ser uma das causas de alteração do funcionamento dos proprioceptores cervicais, podendo ser considerado um fator etiológico de distúrbios do equilíbrio. Essa hipótese tem sido testada em uma variedade de situações ${ }^{4,9}$, no entanto, comprovações mais concretas ainda necessitam ser evidenciadas.

Dessa forma, o objetivo foi avaliar a influência da dor no controle postural de mulheres com dor cervical, verificando sua relação com as possíveis alterações nos sistemas sensoriais e na postura corporal.

\section{PROCEDIMENTOS METODOLÓGICOS}

Trata-se de uma pesquisa exploratória, transversal com análise quantitativa dos dados. O grupo dor cervical foi composto por 29 mulheres, com faixa etária entre 20 e 50 anos, que apresentaram queixas de dor cervical por mais de três meses e sem tratamento médico ou fisioterápico. O grupo controle foi constituído por 20 mulheres sem queixa de dor cervical. Os grupos foram recrutados na comunidade, após divulgação na mídia eletrônica através do site da Instituição, na mídia impressa por meio dos jornais da cidade, por cartazes fixados e junto aos serviços de saúde público e privado da cidade. Foram utilizados como critérios de exclusão: diagnóstico prévio de hérnia de disco e/ou traumas e procedimentos cirúrgicos na coluna vertebral, comprometimentos neurológicos previamente diagnosticados, déficit vestibular e sinais e sintomas de disfunção temporomandibular de grau moderado e grave. O protocolo de pesquisa foi aprovado pelo Comitê de Ética em Pesquisa com Seres Humanos da Universidade Federal de Santa Maria - CAAE $n^{\circ}$ 0329.0.243.000-10. Todos os voluntários formalizaram a participação no estudo, assinando o termo de consentimento livre e esclarecido.

Para a caracterização do grupo dor cervical, utilizou-se uma ficha de anamnese e avaliações clínicas por meio do índice de incapacidade cervical (NeckDisability Index - NDI) e da Escala Visual Analógica (EVA). A intensidade da dor foi avaliada pela EVA que consiste em uma linha horizontal com $10 \mathrm{~cm}$ em que, na extremidade esquerda, encontra-se a indicação sem dor (0) e na direita, dor intensa. O NDI, adaptado e validado para a língua portuguesa, trata-se de um questionário contendo 10 itens elaborados para avaliar a incapacidade e a dor na região da coluna cervical relacionadas às atividades de vida diária. As alternativas numeradas de zero a cinco, descrevem graus crescentes de interferência da dor cervical sobre a realização da atividade questionada. O cálculo do escore final é convertido em um valor percentual, sendo considerados apenas os itens respondidos pelo individuo ${ }^{13}$.

Os dados referentes ao equilíbrio postural foram avaliados por meio de uma plataforma de força AMTI, modelo OR6-6-2000 (AdvancedMechanical 
Technologies, Inc.), que coleta dados referentes ao centro de pressão (COP), com frequência de aquisição de $100 \mathrm{~Hz}$ e tempo de aquisição de 30 segundos, que tem sido sugerido como suficiente para avaliar a oscilação postural ${ }^{11}$ ,principalmente num contexto clínico, onde um minuto ou mais pode ser tempo demais para ficar em pé para o paciente sob análise, que pode levar o indivíduo à fadiga e consequente alteração nos resultados. A plataforma de força mede as variações ocorridas no centro de pressão, definindo a oscilação postural em uma escala de centímetros. As variáveis analisadas foram a amplitude de deslocamento do centro de pressão ântero-posterior (COPap), médio-lateral (COPml) e velocidade média de deslocamento de centro de pressão (COPvel), que são consideradas as principais variáveis utilizadas na investigação do controle postural ${ }^{11}$. A velocidade média de deslocamento do COP foi calculada, dividindo-se o comprimento da trajetória pelo tempo de cada coleta (30s) e o comprimento da trajetória foi calculado pela soma dos deslocamentos entre duas posições subsequentes do COP. A confiabilidade das medidas das variáveis do COP é determinada principalmente por seis principais fatores, frequência de aquisição e de corte dos dados, duração e número de repetições das coletas, condições visuais e superfície $e^{21}$. Os indivíduos foram orientados a se posicionar sobre a plataforma, em posição habitual, pés descalços, cabeça direcionada à frente e manutenção do olhar horizontalizado. As posições dos pés foram marcadas em um papel milimetrado antes da primeira tentativa para que esse posicionamento fosse mantido nas tentativas subsequentes. Foram realizadas três tentativas com os olhos abertos e três tentativas com os olhos fechados. As informações de forças e momentos provenientes da plataforma de força foram tratados em uma rotina desenvolvida no ambiente Interactive Data Linguage (IDL) para a filtragem dos dados e cálculo das variáveis. Foi utilizado um filtro passa-baixa Butterworth, de $4^{\text {a }}$ ordem na frequência de $10 \mathrm{~Hz}$.

Para avaliação do equilíbrio postural com a manipulação dos sistemas somatossensorial, vestibular e visual, foi utilizada a posturografia dinâmica Foam-laser, desenvolvida por Castagno ${ }^{14}$, que expõe o indivíduo a seis tarefas diferentes denominadas Teste de Organização Sensorial (TOS). Os TOS I, II e III foram realizados na posição ortostática habitual, com o mesmo posicionamento dos pés utilizado para a coleta na plataforma. Os TOS IV, V e VI foram realizados na mesma posição anterior, porém, posicionando-se sobre uma almofada de espuma de $10 \mathrm{~cm}$ de espessura de densidade média $\left(0,048 \mathrm{~g} / \mathrm{cm}^{3}\right.$ ou $\left.4,48 \mathrm{~kg} / \mathrm{m}^{3}\right)$, com a finalidade de atenuar a propriocepção. As posições II e V foram realizadas com os olhos fechados. Durante os TOS III e VI, a cabine foi lentamente inclinada, aproximadamente, $20^{\circ}$ para frente, durante 10 s e após retorna a posição inicial em $10 \mathrm{~s}^{15}$ (Figura 1).A análise do sistema somatossensorial é obtida pela divisão do valor do TOS II pelo TOS I, o sistema visual, dividindo-se o valor do TOS IV pelo TOS I, o sistema vestibular, dividindo-se o valor do TOS V pelo TOS I e a preferência visual, dividindo-se o somatório dos valores de TOS III e IV pelo somatório dos valores do TOS II e $\mathrm{V}^{15}$. Para cada teste, os escores foram analisados em 
percentuais, sendo $100 \%$ ausência total de oscilações, diminuindo conforme o aumento de oscilações, chegando a $0 \%$ em casos de quedas. Quanto menor o escore percentual maior o comprometimento dos sistemas.

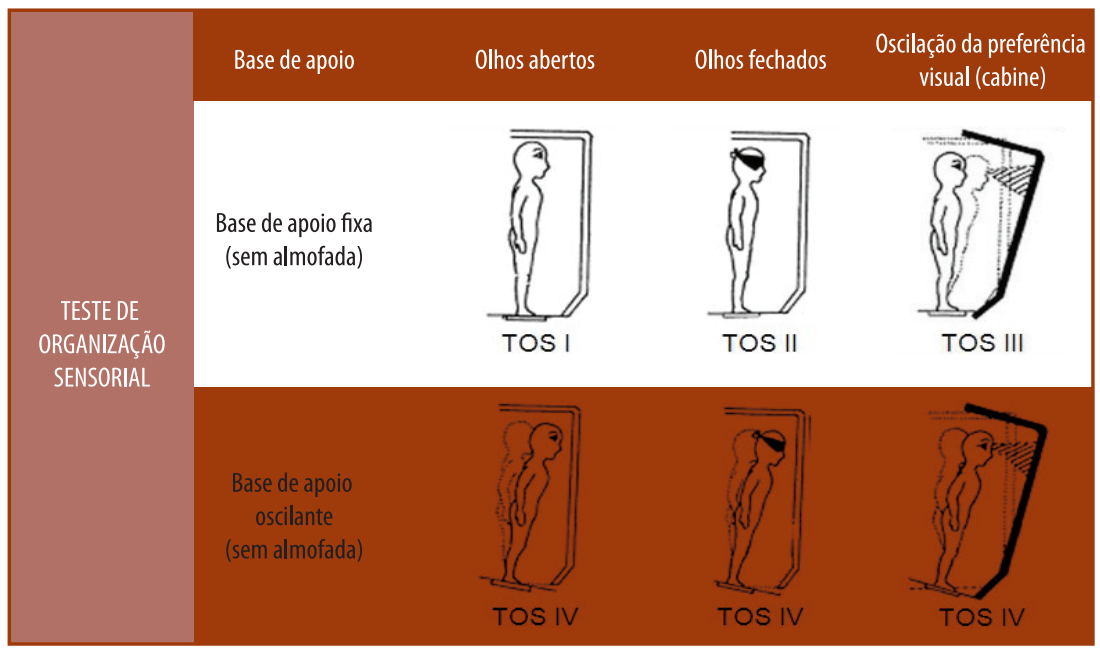

Figura 1. Teste de Organização Sensorial em suas seis condições.

A postura corporal foi avaliada por meio de registro fotográfico com análise biofotogramétrica pelo Software de Avaliação Postural (SAPO) ${ }^{16}$. Estudos atuais ${ }^{16,17}$ demonstraram a confiabilidade e validade nas mensurações de valores angulares dos segmentos corporais.

Para essa pesquisa, analisaram-se as imagens fotográficas no plano sagital direito. Neste plano, optou-se pelo alinhamento horizontal da cabeça (ângulo craniovertebral), alinhamento vertical do tronco, alinhamento horizontal da pelve e ângulo do joelho e tornozelo, que trazem informações tanto do posicionamento dos membros superiores quanto dos membros inferiores. Todos os registros fotográficos foram realizados por um único fotógrafo e os marcadores posicionados pelo mesmo examinador.

Cada procedimento de coleta foi realizado pelo mesmo examinador previamente treinado, cegado para o desfecho do estudo.

Os dados foram submetidos à estatística descritiva, com valores em média e desvio padrão, e estatística analítica. Para verificar a normalidade das variáveis, foi utilizado o teste de Shapiro Wilk. Na comparação entre os grupos, foram utilizados o teste $t$ de student para amostras independentes para as variáveis com distribuição normal e o teste de Mann Whitney para as variáveis com distribuição não-normal. A igualdade das variâncias para as variáveis com distribuição normal foi testada pelo teste de Levene. O nível de significância adotado para todos os testes foi de $5 \%(\alpha=0,05)$.

\section{RESULTADOS}

Os grupos com e sem queixa de dor cervical não apresentaram diferença nas variáveis antropométricas (Tabela 1). Em relação à intensidade da dor 
(EVA), as mulheres sintomáticas apresentaram média de $5,38 \pm 0,53$ pontos e no índice de incapacidade cervical (NDI), escores entre 21-40\% indicando déficit moderado.

Tabela 1. Variáveis antropométricas, intensidade da dor e incapacidade cervical.

\begin{tabular}{lccc}
\hline \multirow{2}{*}{ Variáveis } & Grupo dor cervical $(\mathrm{n}=29)$ & Grupo controle $(\mathrm{n}=20)$ & \multirow{2}{*}{ p-valor } \\
\cline { 2 - 3 } & Média $\pm \mathrm{DP}$ & \multicolumn{1}{c}{ Média $\pm \mathrm{DP}$} & \\
\hline Idade (anos) & $32,21 \pm 10,48$ & $28,30 \pm 6,24$ & 0,51 \\
Estatura $(\mathrm{m})$ & $1,65 \pm 0,07$ & $1,64 \pm 0,08$ & 0,51 \\
Massa $(\mathrm{kg})$ & $61,65 \pm 10,12$ & $59,63 \pm 11,01$ & 0,61 \\
IMC $\left(\mathrm{kg} / \mathrm{m}^{2}\right)$ & $22,07 \pm 3,05$ & $22,20 \pm 3,12$ & 0,27 \\
\hline
\end{tabular}

Legenda: IMC = Índice de Massa Corporal.

Nas variáveis de equilíbrio postural, as mulheres com dor cervical apresentaram maior oscilação (Tabela 2).

Tabela 2. Variáveis da plataforma de força relacionadas ao centro de pressão.

\begin{tabular}{lccc}
\hline \multirow{2}{*}{ Variáveis } & Grupo Dor cervical $(\mathrm{n}=29)$ & Grupo Controle $(\mathrm{n}=20)$ & \multirow{2}{*}{ p-valor } \\
\cline { 2 - 3 } & Média \pm DP & Média $\pm \mathrm{DP}$ & \\
\hline CopAP OA $(\mathrm{cm})$ & $2,22 \pm 0,89$ & $1,13 \pm 0,18$ & $<0,01^{*}$ \\
CopML OA $(\mathrm{cm})$ & $1,35 \pm 0,67$ & $0,76 \pm 0,17$ & $<0,01^{*}$ \\
CopVel OA $(\mathrm{cm} / \mathrm{s})$ & $0,96 \pm 0,22$ & $0,72 \pm 0,16$ & $<0,01^{*}$ \\
CopAP OF $(\mathrm{cm})$ & $2,68 \pm 1,07$ & $1,47 \pm 0,27$ & $<0,01^{*}$ \\
CopML OF $(\mathrm{cm})$ & $1,51 \pm 0,65$ & $0,89 \pm 0,24$ & $<0,01^{*}$ \\
CopVel OF $(\mathrm{cm} / \mathrm{s})$ & $1,13 \pm 0,33$ & $0,91 \pm 0,23$ & $0,03^{*}$ \\
\hline
\end{tabular}

Legenda: $\mathrm{AO}=$ olhos abertos; $\mathrm{OF}=0$ lhos fechados; $\mathrm{COPap}=$ amplitude de deslocamento ântero-posterior, COPml = amplitude de deslocamento médio-lateral do centro de pressão e COPvel = velocidade média de deslocamento de centro de pressão. *Mann Whitney, $p<0,05$.

Nas variáveis de equilíbrio postural estudadas, observou-se uma maior amplitude e velocidade de deslocamento do centro de pressão no grupo dor cervical. Nas duas condições de teste, olhos abertos e fechados, as diferenças encontradas foram significativas, demonstrando maior oscilação postural no grupo com dor cervical. Em relação aos critérios de confiabilidade das medidas das variáveis do COP, poucos estudos cumprem com a metade dos critérios recomendados, sendo que, neste estudo, foram respeitados 5 dos 6 critérios de confiabilidade.

$\mathrm{Na}$ avaliação dos segmentos corporais, as mulheres com dor cervical apresentaram anteriorização da cabeça (Tabela 3). Na avaliação postural, os grupos se diferenciaram significativamente apenas no ângulo craniovertebral, que foi menor nas mulheres sintomáticas, demonstrando uma anteriorização do segmento cefálico.

Em relação aos dados provenientes da posturografia dinâmica Foam-laser, observou-se diferença estatística entre os dois grupos nas seis situações de teste. $\mathrm{O}$ escore obtido pelo grupo dor cervical, nas seis condições sensoriais, demonstrou que o equilíbrio postural apresentou maior comprometimento (Tabela 4). 
Tabela 3. Variáveis angulares utilizadas para a avaliação postural.

\begin{tabular}{llll}
\hline \multirow{2}{*}{ Variáveis } & Grupo dor cervical $(\mathrm{n}=29)$ & Grupo controle $(\mathrm{n}=20)$ & \\
\cline { 2 - 4 } & Média $\pm \mathrm{DP}$ & Média $\pm \mathrm{DP}$ & $\mathrm{p}$-valor \\
\hline Ângulo CV $\left(^{\circ}\right)$ & $48,53 \pm 4,37$ & $52,17 \pm 3,93$ & $<0,01^{* *}$ \\
\hline Alinhamento do corpo $\left(^{\circ}\right)$ & $-0,11 \pm 2,70$ & $-0,19 \pm 2,01$ & 0,80 \\
Alinhamento da pelve $\left(^{\circ}\right)$ & $-11,19 \pm 7,36$ & $-7,03 \pm 5,85$ & 0,07 \\
Ângulo do joelho $\left(^{\circ}\right)$ & $0,08 \pm 3,40$ & $0,16 \pm 2,63$ & 0,93 \\
Ângulo do tornozelo $\left(^{\circ}\right)$ & $85,35 \pm 2,10$ & $85,41 \pm 2,24$ & 0,92 \\
\hline
\end{tabular}

Legenda: $\mathrm{CV}=$ crâniovertebral. Nível de significância: **Mann Whitney, $\mathrm{p}<0,05$. Valores expressos em graus $\left({ }^{\circ}\right)$.

Tabela 4. Comparação dos Testes de Organização Sensorial entre os grupos com queixa de dor cervical e controle.

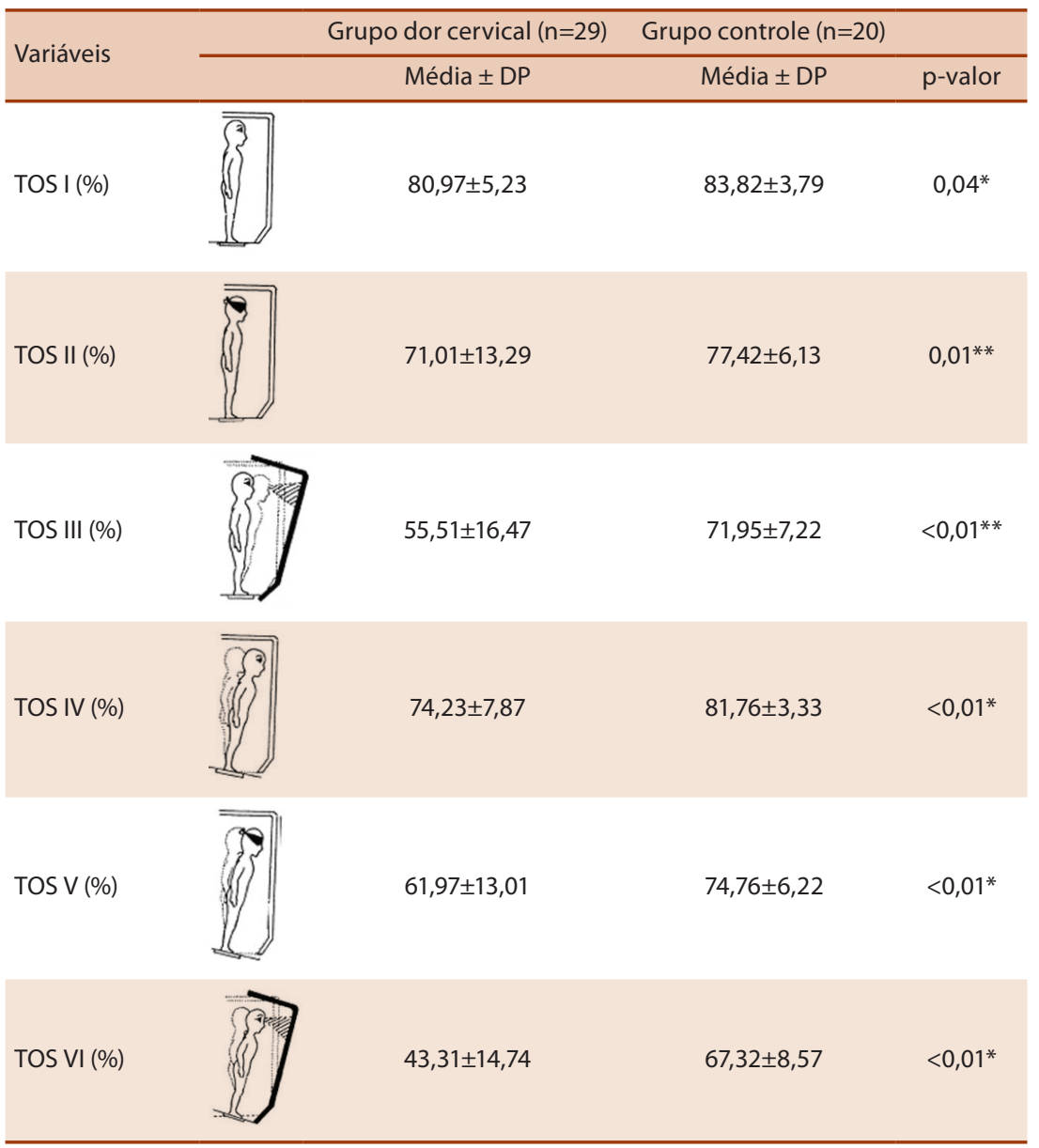

Legenda: TOS = Teste de organização sensorial; ${ }^{*} t$-student para amostras independentes, $\mathrm{p}<0,05 ;{ }^{* *}$ Mann Whitney, $\mathrm{p}<0,05$.

$\mathrm{Na}$ avaliação dos sistemas sensoriais, o grupo com dor cervical apresentou valores percentuais menores. Os sistemas visual, vestibular e o preferencial apresentaram diferença significativa na comparação entre os grupos. Apesar de valores inferiores, apenas o sistema somatossensorial não apresentou diferença significativa. 


\section{DISCUSSÃO}

O objetivo desse estudo foi avaliar a influência da dor no controle postural de mulheres com dor cervical, verificando sua relação com as possíveis alterações nos sistemas sensoriais e na postura corporal comparando com mulheres assintomáticas. Os resultados demonstraram que, para este grupo de estudo, as mulheres com dor cervical apresentaram maior oscilação postural e maior comprometimento dos sistemas sensoriais nas condições testadas sugerindo um déficit no equilíbrio postural.

Todas as variáveis relacionadas ao COP apresentaram diferença significativa na comparação entre os dois grupos nas duas condições testadas, demostrando que as mulheres com dor não específica na região cervical apresentaram significativa instabilidade postural. Revisão sistemática recente ${ }^{18}$ mostrou que na maioria dos estudos houve variabilidade nas medidas do COP, indicando que indivíduos com dor cervical apresentam instabilidade postural quando comparados a controles com diferença significativa na dor por lesão em chicote induzida por trauma. No entanto, poucos estudos ${ }^{4,19}$ encontraram diferença significativa quando indivíduos com dor cervical não específica foram comparados a controles.

Dentre as variáveis mensuradas para a oscilação postural, o COPvel tem se mostrado um parâmetro consistente ${ }^{20}$ e de alta confiabilidade com valores discriminativos em condições de dor, sugerindo que esta relação deve ser investigada em pacientes com dor cervical ${ }^{18}$. Neste estudo, esta variável foi investigada, mostrando que mulheres com dor cervical apresentaram maior COPvel nas duas condições de teste, olhos abertos e fechados, sugerindo aumento da oscilação postural. Da mesma forma, Vuillerme et al. ${ }^{(19)}$ e Endo et al. ${ }^{21}$ utilizaram esta variável, encontrando diferença significativa na condição olhos abertos ${ }^{19,21}$ e fechados ${ }^{20}$,quando compararam indivíduos com cervicalgia e controle.

Em relação à postura corporal, os grupos não apresentaram diferença estatística nas medidas angulares, exceto no ângulo craniovertebral (CV), demonstrando uma postura anteriorizada da cabeça nas mulheres com queixa de dor cervical.

Há evidências de que a manutenção da postura anteriorizada da cabeça resulta em aumento das cargas compressivas sobre a coluna cervical e deformação dos tecidos ${ }^{22}$. Chiu et al. ${ }^{23}$ e Silva et al. ${ }^{24}$ encontraram correlação negativa entre o ângulo CV e a intensidade da dor, sugerindo que quanto maior a anteriorização da cabeça maior possibilidade de dor cervical. Os resultados deste estudo mostraram que o ângulo CV foi significativamente menor nas mulheres com dor cervical, demonstrando que estas apresentaram uma postura anteriorizada da cabeça. Esses achados podem ser explicados pelo fato de que a manutenção da postura anteriorizada da cabeça sobrecarrega as estruturas não contráteis e aumenta a tensão muscular nas estruturas cervicais posteriores, gerando a dor miofascial ${ }^{23}$.

A ocorrência dessa dor pode causar aumento da inibição pré-sináptica da musculatura aferente, bem como afetar a modulação central dos fusos 
proprioceptivos do músculo causando latências prolongadas ${ }^{25,26}$. Estas alterações podem levar a uma diminuição do controle muscular, resultando em aumento da oscilação postural ${ }^{18}$. A intensidade da dor tem sido considerada fator determinante na avaliação da oscilação postural ${ }^{18}$. Indivíduos com baixa intensidade de dor cervical não apresentaram diferença significativa na oscilação postural ${ }^{4}$, enquanto que indivíduos com maior intensidade de dor diferenciaram-se significativamente dos controles ${ }^{19}$.

Da mesma forma, a deterioração da informação proprioceptiva da região cervical pode ser considerada um fator determinante na diminuição da acurácia do processo de integração sensoria ${ }^{18}$. Além disso, maiores intensidades de dor e déficits neurológicos ou vestibulares podem ser considerados fatores determinantes no aumento da oscilação postural ${ }^{18}$. Neste estudo, déficits neurológicos e vestibulares foram considerados critérios de exclusão, no entanto, as mulheres com dor cervical apresentaram déficit do equilíbrio postural sugerindo comprometimento dos sistemas vestibular, visual e somatossensorial.

Na posturografia, as principais condições que avaliam a função do sistema vestibular são aquelas que permitem a oscilação perante uma superfície móvel, neste caso, a utilização da almofada, obtida nas condições TOS IV, V e VI. Nessas situações, a oscilação fisiológica do centro de gravidade do corpo desloca a superfície de apoio com a oscilação corporal e obriga o indivíduo a utilizar a informação vestibular para os ajustes compensatórios, evitando a queda ${ }^{27}$. Nessa condição, o sistema vestibular é a aferência cuja precisão da informação tem o maior impacto na estabilização corporal, pois as informações vestibulares são as mais fiéis diante de um ambiente que oferece informações conflitantes quando comparadas às informações visuais e proprioceptivas ${ }^{28}$.

Neste estudo, as mulheres sintomáticas apresentaram menores índices em todos os sistemas sensoriais avaliados, sendo importante destacar que, além da presença de dor, o aumento da oscilação postural neste grupo de mulheres se deve a um maior conflito na integração das informações oriundas dos três sistemas envolvidos, mesmo mantendo a proporção do sistema visual, seguido pelo proprioceptivo e por último o vestibular. Esse achado pode ser explicado pela importância da cabeça para a manutenção do controle postural, pois acomoda dois dos principais órgãos sensoriais, o labirinto e o órgão visual ${ }^{29}$.

Alterações no posicionamento cefálico podem comprometer a resposta eferente que o SNC emite ao complexo neuromuscular por meio dos reflexos vestíbulo-espinhal e vestíbulo-ocular, causando alterações nas relações ópticas, no posicionamento vestibular e alteração na propriocepção cervical ${ }^{30}$. Tendo em vista essa possível relação entre o posicionamento cefálico e os sistemas sensoriais sugere-se, para os próximos estudos, a realização da vectoeletronistagmografia para avaliação da componente vestibular a fim de descartar o comprometimento vestibular nestes pacientes.

Sugere-se que os profissionais envolvidos na reabilitação de indivíduos com dor cervical estejam cientes da possivel relação entre a postura 
anteriorizada da cabeça, presença de dor e oscilação postural. O enfoque multidisciplinar deve levar em consideração a reeducação postural como parte integrante da prevenção e reabilitação de pacientes com dor cervical, assim como, a avaliação dos sistemas sensoriais em diferentes condições de teste para melhorar o direcionamento da reabilitação.

\section{CONCLUSÃO}

A dor cervical e a postura anteriorizada do segmento cefálico têm efeito deletério no controle postural de mulheres sintomáticas, tanto na postura estática quanto na postura dinâmica.

\section{REFERÊNCIAS BIBLIOGRÁFICAS}

1. Kapreli E, Vourazanis E, Billis E, Oldham JA, Strimpakos N. Respiratory dysfunction in chronic neck pain patients A pilot study. Cephalalgia 2009;29:701-10.

2. Hogg-Johnson S, Van der Velde G, Carroll LJ, Holm LW, Cassidy JD, Guzman J et al. The burden and determinants of neck pain in the general population: results of the Bone and Joint Decade 2000-2010 Task Force on Neck Pain and Its Associated Disorders. Spine 2008;33(4S):S39-S51.

3. Carroll LJ, Hogg-Johnson S, Van der Velde G, Haldeman S, Holm LW, Carragee EJet al. Course and prognostic factors for neck pain in the general population: results of the Bone and Joint Decade 2000-2010 Task Force on Neck Pain and Its Associated Disorders. J Manipulative Physiol Ther 2009;32(2S):S87-S96.

4. Field S, Treleaven J, Jull G. Standing Balance: A comparison between idiopathic and whiplash-induced neck pain. Man Ther 2008;13:183-91.

5. Jørgensen MB, Skotte JH, Holtermann A, Sjøgaard G, Petersen NC, Søgaard K. Neck pain and postural balance among workers with high postural demands - a cross-sectional study. BMC Musculoskelet Disord 2011;12:176.

6. Armijo Olivo S, Magee DJ, Parfitt M, Major P, Thie NMR. The association between the cervical spine, the stomatognathic, system and craniofacial pain: a critical review. J Orofac Pain 2006;20(4):271-87.

7. Bevilaqua-Grossi D, Chaves TC, Oliveira AS. Cervical Spine signs and symptons: perpetuating rather than predisposing factors for temporomandibular disorder in women. J Appl Oral Sci 2007;15(4):259-64.

8. Karlberg M, Persson PT, Magnusson M. Reduced postural control in patients with chronic cervicobrachial pain syndrome. Gait Posture 1995;3:241-49.

9. Giacomini PG, Alessandrini M, Evangelista M, Napolitano B, Lanciani R, Camaioniet D. Impaired postural control in patients affected by tension-type headache. Eur J Pain 2004;8(6):579-83.

10. Ionescu E, Morletb T, Froehlichc P, Ferber-Viartdet C. Vestibular assessment with Balance Quest Normative data for children and young adults. Int J Pediatr Otorhinolaryngol 2006;70:1457-65.

11. Duarte M, Freitas SMSF. Revisão sobre posturografia baseada em plataforma de força para avaliação do equilíbrio. Rev bras fisioter 2010;14(3):183-92.

12. Mochizuki, L.; Amadio C. A. As informações sensoriais para o controle postural. Fisioter Mov 2006;19(2):11-8.

13. Vernon H, Mior S. The Neck Disability Index: a study of reliability and validity. J Manipulative Physiol Ther 1991;14(7):409-15.

14. Castagno LA. A new method for sensory organization tests: the foam-laser dynamic posturography. Rev Bras Otorrinolaringol 1994;60(4):287-96.

15. Loth EA, Albuquerque CA, Ciena AP, Rossi AG. Avaliação do Controle Postural em Adultos Jovens Através da Posturografia Dinâmica Foam-Laser e Plataforma de Força. Rev Bras Med Esporte 2011;17(3):171-4. 
16. Ferreira EAG. Postural assessment software (PAS/SAPO): validation and reliability. Clinics 2010;65(7):675-81.

17. Braz RG, Goes FPC, Carvalho GA. Confiabilidade e validade de medidas angulares por meio do software para avaliação postural. Fisioter Mov 2008;21(3):117-26.

18. Ruhe A, Fejer R, Walker B. Altered postural sway in patients suffering from non-specific neck pain and whiplash associated disorder - A systematic review of the literature. Chiropr Man Therap 2011;19:13.

19. Vuillerme N, Pinsault N. Experimental neck muscle pain impairs standing balance in humans. Exp Brain Res 2009;192:723-9.

20. Ruhe A, Fejer R, Walker B. The test-retest reliability of centre of pressure measures in bipedal static task conditions - A systematic review of the literature. Gait Posture 2010;32:436-45.

21. Endo K, Suzuki H, Yamamoto K. Consciously postural sway and cervical vertigo after whiplash injury. Spine 2008;33:E539-42.

22. Bonney RA, Corlett EN. Head posture and loading of the cervical spine. Appl Ergon 2002; 33:415-7.

23. Chiu TTW, Ku WY, Lee MH, Sum WK, Wan MP, Wong CY et al. A study on the prevalence of and risk factors for neck pain among university academic staff in Hong Kong. J Occup Rehabil 2002; 12:77-91.

24. Silva AG, Punt TD, Sharples P, Vilas-Boas JP, Johnson MI. Head posture and neck pain of chronic nontraumatic origin: a comparison between patients and pain-free persons. Arch Phys Med Rehabil 2009;90:669-74

25. Sibley KM, Carpenter MG, Perry JC, Frank JS. Effects of postural anxiety on the soleus H-reflex. Hum Mov Sci 2007;26:103-12.

26. Capra NF, Ro JY. Experimental muscle pain produces central modulation of proprioceptive signals arising from jaw muscle spindles. Pain 2000;86:151-62.

27. Teixeira CS, Körbes D, Rossi AG. Ruído e equilíbrio: aplicação da posturografia dinâmica em indústria gráfica. Rev CEFAC 2011;13(1):92-101.

28. Loth EA, Rosi AG, Cappellesso PC, Ciena AP. Avaliação da influência do sistema vestibular no equilíbrio de adultos jovens através de posturografia dinâmica foam-laser e plataforma de força. Semina Cienc Biol Saúde 2008; 29(1):57-64.

29. Maisson J. Postural control systems in developmental perspective. Neurosci Biobehav Rev 1998; 22(4):465-72.

30. Treleaven J. Sensorimotor disturbances in neck disorders affecting postural stability, head and eye movement control. Man Ther 2008;13:2-11.

Endereço para correspondência

Juliana Corrêa Soares Rua Appel, 1405/302 CEP: $97015-030$

Santa Maria, RS. Brasil

E-mail: jjuzinha83@yahoo.com.br 